\title{
Proyecto de implantación de un programa de educacion para la salud en el proceso asistencial del paciente trasplantado renal
}

\author{
M' José Arnau Vives* \\ Mar Rueda Jarque** \\ Marisa Vilplana Molto** \\ Celsa Pernas Pérez**
}

Servicio de Nefrología y $H D$

Hospital General Vall d'Hebron (Barcelona)

* Supervisora

** DUE

\section{RESUMEN}

En la actualidad, una de las funciones relevantes del rol de enfermería es la docente y educadora, realizando promoción y educación para la salud de la población a través de una potenciación del autocuidado.

A través de nuestra práctica asistencial hemos detectado la necesidad de implantar un programa de educación para la salud en el paciente trasplantado renal, con el objetivo de prevenir las complicaciones derivadas del tratamiento, de la adherencia al tratamiento y déficit de autocuidados asociados al trasplante renal.

Para definir los objetivos de nuestro programa nos hemos basado en el método PRECEDE, uno de los más utilizados en educación sanitaria. La duración del estudio será de un año, llevado a la práctica por el equipo de Enfermería de la Unidad de Nefrología. Se realizarán charlas informativas, entregándose folletos. La evaluación se hará a partir de dos test, uno para valorar los conocimientos adquiridos por los pacientes y otro para valorar la metodología utilizada por enfermería y el contenido didáctico de las charlas y folletos.

\begin{tabular}{|c|}
\hline Correspondencia: \\
Mª José Arnau Vives \\
Servicio de Nefrología, Planta 7, anexo pares \\
Hospital General Vall d'Hebron \\
Avda. Vall d'Hebron, 119-129 \\
08035 Barcelona \\
Email: mjarnau@cs.vhebron.es
\end{tabular}

Con la implantación de este programa queremos disminuir la ansiedad del paciente derivada de la falta de información y conocimientos, así como los reingresos debidos al déficit de autocuidados.

$\begin{array}{ll}\text { PALABRAS CLAVE: } & \text { EDUCACIÓN SANITARIA } \\ & \text { MÉTODO PRECEDE } \\ & \text { TRASPLANTE RENAL } \\ & \text { AUTOCUIDADO }\end{array}$

\section{PROJECT FOR THE IMPLEMENTATION OF A HE- ALTH EDUCATION PROGRAMME IN THE CARE OF RENAL TRANSPLANT PATIENTS}

\section{SUMMARY}

At present, one of the relevant functions of the nursing role is teaching and educating, carrying out health promotion and education tasks for the population by encouraging self care.

In the course of our clinical practice, we have detected the need to implement a health education programme for renal transplant patients, with the aim of preventing complications arising as a result of the treatment, adherence to treatment and lack of self care associated with renal transplants.

To define the aims of our programme, we have used the PRECEDE method, one of the methods most widely used in health education. The duration of the study will be one year, and will be carried out by the Nursing team of the Nephrology Unit. 
Talks will be organized, at which leaflets will be handed out. The evaluation will be carried out on the basis of two tests, one to evaluate the knowledge acquired by patients and another to evaluate the methodology used by nursing staff and the educational content of the talks and leaflets.

With the implementation of this programme, we want to reduce patient anxiety caused by a lack of information and knowledge, as well as the number of re-admissions due to deficient self care.

\section{KEY WORDS: HEALTH EDUCATION \\ PRECEDE METHOD \\ RENAL TRANSPLANT \\ SELF CARE}

\section{INTRODUCCIÓN Y OBJETIVO}

El principio fundamental del ciudadano a tener información sobre salud le empujan, en muchos casos, a autoresponsabilizarse de su propia salud ${ }^{(1)}$. El ciudadano actual cada vez demanda más información sobre cuidados de salud, ya sea buscando el consejo profesional o a través de otras fuentes. Para llegar a un buen grado de autoresponsabilidad es necesario partir de la información adecuada, bien canalizada y acorde con la comprensión del destinatario.

Virginia Henderson definió los cuidados de enfermería como "la capacidad de asistir al individuo, enfermo o sano, en la realización de actividades que contribuyen a su salud o a su recuperación, y que él realizaría sin ayuda si dispusiera de la fuerza, voluntad o conocimientos necesarios, de tal forma que le ayude a ser independiente lo antes posible"(2). En la actualidad, una de las funciones relevantes del rol de Enfermería es la docente y educadora, realizando promoción y educación para la salud de la población, a través de una potenciación del autocuidado.

La educación para la salud (EpS) tiene dos objetivos fundamentales: capacitar a las personas para tomar decisiones informadas que favorezcan la salud y conseguir su participación en el proceso educativo mediante una actitud crítica y una implicación en las decisiones que favorezcan la salud ${ }^{(3)}$. Con la EpS, lo que se pretende es mejorar la salud y el bienestar de los individuos; se intenta transmitir conocimientos, hábitos de vida y actitudes saludables.

El hecho que el trasplante renal sea el único tratamiento sustitutivo capaz de devolver completamente la función renal nor- mal hace que los pacientes tengan grandes expectativas en la realización de un trasplante. Es de primordial importancia informarle que necesitará seguir un tratamiento, control y atención profesional permanente. El paciente trasplantado y su familia precisan adquirir conocimientos sobre el tratamiento farmacológico, la alimentación adecuada, el reconocimiento de síntomas y signos de alarma, y todos aquellos factores que le permitan realizar un autocuidado de calidad, ya que una buena adherencia al tratamiento y control puede disminuir el riesgo de complicaciones, de reingresos hospitalarios y de la pérdida del injerto renal.

A través de la práctica asistencial y realizando un seguimiento de los pacientes trasplantados que reingresaban en nuestra unidad debido a complicaciones, el personal de enfermería del Servicio de Nefrología detectó que, en algunos casos, había un déficit de conocimientos sobre los cuidados a realizar en domicilio tras el trasplante y que había que reforzar y ampliar la información proporcionada. Por este motivo, nos planteamos crear un programa de EpS, proporcionando una información consensuada por el equipo, que diera respuesta a las necesidades de información del paciente trasplantado renal.

Para definir los objetivos de nuestro programa nos basamos en el método PRECEDE, uno de los modelos de planificación más usados en educación sanitaria, que permite establecer un diagnóstico educativo, al analizar los diferentes factores que determinan la puesta en práctica de un comportamiento ${ }^{(4-9)}$.

Según este método el objetivo general describe el estado de salud que queremos alcanzar. Los objetivos intermedios definen los comportamientos esperados en la población. Los objetivos específicos favorecen la predisposición, la facilitación y el refuerzo del cambio de comportamiento que se quiere conseguir. Se trabajan las áreas de conocimientos, habilidades y actitudes (figura 1).

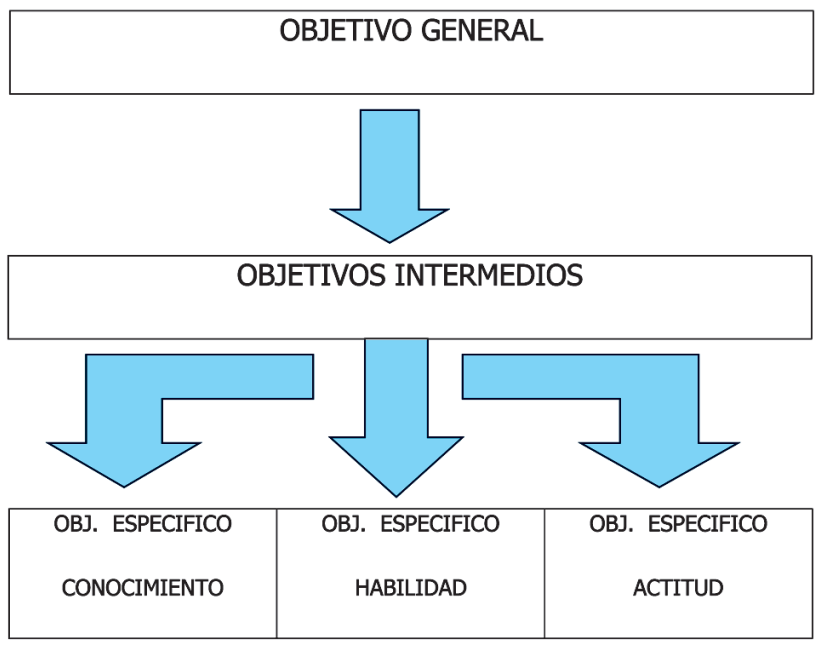

Figura 1. Diagrama para planificación y evaluación del programa. 
El objetivo general que nos planteamos en nuestro programa de EpS es prevenir las complicaciones del trasplante renal asociadas a la incorrecta adherencia al tratamiento y déficit de autocuidados. Este objetivo lo hemos desglosado en ocho objetivos intermedios, y éstos a su vez en tres objetivos específicos, que trabajan las áreas de conocimientos, habilidades y actitudes del paciente.

\section{MATERIAL Y MÉTODO}

El programa de EpS será llevado a cabo por el equipo de enfermería de Nefrología del Hospital Vall d'Hebron (Barcelona), Se iniciará en enero de 2005, Incluiremos en el programa a todos los pacientes trasplantados en la unidad de trasplante renal en el período de un año, informándoles del programa e invitándoles a participar, según las normas de nuestro hospital ${ }^{(5)}$.

Hemos realizado un plan educativo, basándonos en la bibliografía consultada ${ }^{(3)}$, el cual incluye un contenido didáctico, la metodología que se va a emplear para presentarlo y el cronograma a realizar según vía clínica en el proceso del trasplante renal sin complicaciones (+/-10 días desde el ingreso hasta el alta hospitalaria).

El contenido didáctico es la información que proporcionaremos al paciente e incluye los siguientes temas que son relevantes en el trasplante renal ${ }^{(6-7)}$ : medicación inmunosupresora, alimentación tras el trasplante renal, hábitos saludables y autocuidados, dudas que pueden surgir, autocontroles domiciliarios y complicaciones médicas más frecuentes.

Presentaremos esta información al paciente a través de charlas individualizadas y de corta duración, realizadas en la propia habitación a partir del momento en que su estado físico y emocional lo permitan, y durante toda la estancia hospitalaria. Entregaremos un folleto informativo que contendrá todos los temas del contenido didáctico, elaborado por el personal de enfermería de Nefrología y editado por la Unidad de Comunicación e Imagen del hospital. La enfermera responsable de los pacientes de la unidad de trasplante renal será la encargada de proporcionar la información al paciente mientras esté ingresado.

También hemos realizado dos test, uno de conocimientos y otro de retroacción (anexo 1), para realizar la evaluación del programa. Hemos desarrollado unos esquemas para la planificación y evaluación del programa, siguiendo ocho objetivos intermedios, los cuales nos ayudarán a organizar la presentación del contenido didáctico y a evaluar si los objetivos planteados han sido alcanzados. Estos esquemas garantizan una atención continuada por parte de todo el equipo de enfermería según el cronograma preestablecido en el plan educativo. A modo de ejemplo presentamos el esquema del primer objetivo intermedio de nuestro programa de educación (anexo 2).

\section{DISCUSIÓN}

La evaluación es un componente integral de la educación para la salud y es necesaria para valorar su efectividad. El proceso de educación debe ser evaluado tanto por la enfermera como por el paciente. La enfermera debe remitirse a los objetivos planteados para realizar la evaluación ${ }^{(3)}$.

El test de evaluación de conocimientos será realizado por Enfermería y nos servirá para conocer el grado de instrucción del paciente. Para conocer si la metodología de intervención y el contenido didáctico han sido satisfactorios para los pacientes, y saber si tendríamos que plantearnos cambios en nuestra estrategia educativa, hemos considerado oportuno realizar un test de retroacción. Este test se realizará en consultas externas, una vez el paciente sea dado de alta hospitalaria.

Esperamos que la EpS basada en la planificación de estrategias de adquisición de conocimientos, habilidades y actitudes por parte del paciente, con el fin de potenciar nuevos hábitos de salud, permitirá la unificación de criterios de actuación y la evaluación por parte de Enfermería desde el primer día del ingreso del paciente, hasta su alta hospitalaria.

Con la implantación del programa de educación para la salud queremos disminuir la ansiedad en el paciente derivada de la falta de información y conocimientos, así como los reingresos derivados del déficit de autocuidados.

\section{BIBLIOGRAFÍA}

1. Ley $41 / 2002,14$ noviembre, básica y reguladora de la autonomía del paciente y de derechos y obligaciones en materia de información y documentación clínica.

2. Kozier. Biblioteca Enfermería Profesional. Tomo 1, Enfermeria Fundamental. Interamericana-McGraw-Hill.

3. Cheeseman GS, Selekman J, Belza Tack B, Parker Martin J. Manual de Educación Sanitaria del Paciente. Ediciones Doyma.

4. Guayta Escolies R, Isla Pera P. Determinantes de la conducta: teorías científicas sobre la modificación de comportamientos. Unidad didáctica 4.

5. Recomendaciones del CEA. El proceso de información en el consentimiento informado. Grupo de trabajo Consentiment informat. Hospital Vall d'Hebron. Julio 2003. 
6. Andreu Periz L, Force Sanmartín E, Campistol Plana JM. El Trasplante Renal: una nueva forma de vida. DRUG FARMA. 2000.

7. Lloveres Macià J, Masramon Fontanals J, Arubia Marimon J, Mir Fontana M, Orfila Gornés A, De las Cuevas Bou X, Cordovilla Bilbao L. Manual del Paciente Trasplantado Renal. Institut Català de la Salut.
8. Kuss R, Bourget P. Una Historia Ilustrada del Trasplante de Órganos. Sandoz. 1992.

9. Bimbela JL, Gomez Rodríguez C. Sida y comportamientos preventivos: el modelo PRECEDE. Rev de Psicología General y Aplicada 1994; 47(2):151-157.

\section{Anexo 1}

\section{Test de retroación}

Por favor, coloque una cruz en el número que corresponda a la evaluación que usted considere a cerca de su aprendizaje

EXCELENTE: 5; MUY BUENA: 4; BUENA: 3; REGULAR: 2; MALA: 1

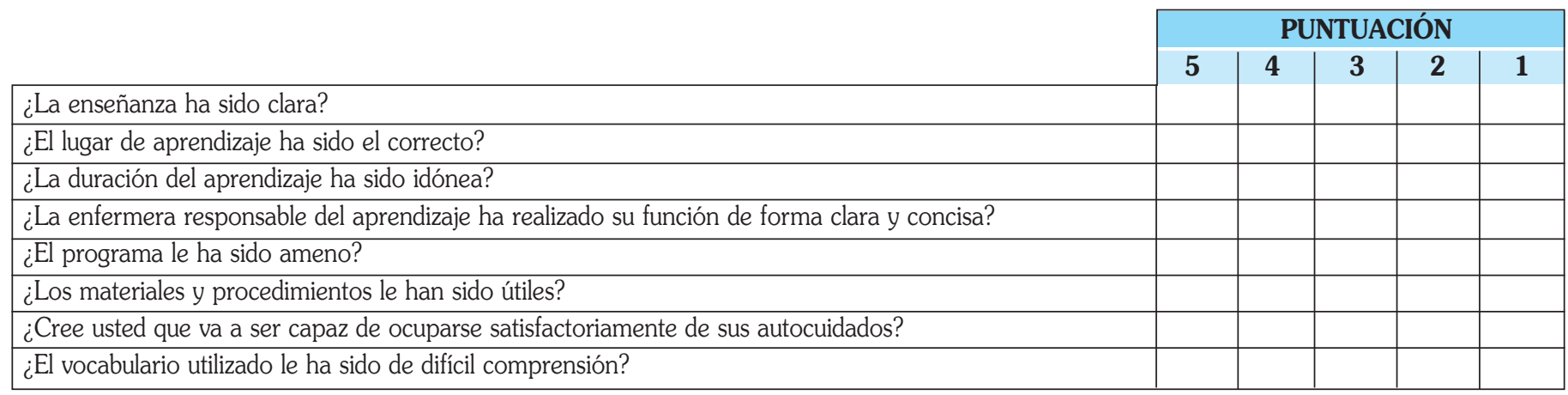

¿Desea añadir algún comentario en relación a su programa formativo, con el objetivo de ayudarnos a mejorarlo? .....

\section{Anexo 2}

\section{ORGANIZACIÓN Y EVALUACIÓN DEL PROGRAMA DE EDUCACIÓN PARA LA SALUD DEL PACIENTE TRASPLANTADO RENAL 1er OBJETIVO INTERMEDIO \\ - Realizarán correctamente el tratamiento farmacológico}

\begin{tabular}{|c|c|c|c|c|c|c|}
\hline Objetivo especifico & Contenido & $\begin{array}{c}\text { Ejecución: firma } \\
\text { y fecha }\end{array}$ & Actividad & Material & $\begin{array}{l}\text { Método de } \\
\text { evaluación }\end{array}$ & $\begin{array}{l}\text { Evaluación: } \\
\text { fecha y firma }\end{array}$ \\
\hline $\begin{array}{l}\text { Conocimientos: } \\
\text { Cocerán la dosis, } \\
\text { pauta de administración, } \\
\text { propiedades y efectos } \\
\text { secundarios de la medicación }\end{array}$ & $\begin{array}{l}\text { Nociones elementales } \\
\text { sobre la medicación. }\end{array}$ & & $\begin{array}{l}\text { Charla, información verbal } \\
\text { apoyada por información } \\
\text { escrita }\end{array}$ & $\begin{array}{c}\text { Información escrita } \\
\text { Manual del trasplantado } \\
\text { renal. }\end{array}$ & $\begin{array}{l}\text { Cuestionario. } \\
\text { Expresarán } \\
\text { conocimientos sobre } \\
\text { su medicación }\end{array}$ & $\begin{array}{l}\text { Objetivo: } \\
\text { Alcanzado } \\
\text { No alcanzado } \\
\text { Firma: }\end{array}$ \\
\hline $\begin{array}{c}\text { Habilidades: } \\
\text { Serán capaces de } \\
\text { autoadministrarse } \\
\text { correctamente la } \\
\text { medicación } \\
\text { (dosis, horarios...) }\end{array}$ & $\begin{array}{l}\text { Explicar cómo tomar } \\
\text { correctamente la } \\
\text { medicación y su } \\
\text { importancia }\end{array}$ & & $\begin{array}{c}\text { Información verbal } \\
\text { y escrita }\end{array}$ & $\begin{array}{l}\text { Hoja explicativa de la } \\
\text { toma de medicación. } \\
\text { Información } \\
\text { proporcionada por } \\
\text { Farmacia }\end{array}$ & $\begin{array}{c}\text { Cuestionario. } \\
\text { Responderán qué dosis } \\
\text { y pauta de administración } \\
\text { han de seguir } \\
\text { en su domicilio. }\end{array}$ & $\begin{array}{l}\text { Objetivo: } \\
\text { Alcanzado } \\
\text { No alcanzado } \\
\text { Firma: }\end{array}$ \\
\hline $\begin{array}{c}\text { Actitudes: } \\
\text { Valorarán los riesgos } \\
\text { que comporta no tomar la } \\
\text { medicación adecuadamente }\end{array}$ & $\begin{array}{l}\text { Enumerar los efectos } \\
\text { adversos en la no correcta } \\
\text { toma de medicación. }\end{array}$ & & $\begin{array}{c}\text { Información verbal } \\
\text { y escrita }\end{array}$ & $\begin{array}{l}\text { Información escrita. } \\
\text { Manual del } \\
\text { trasplantado renal. }\end{array}$ & $\begin{array}{l}\text { Cuestionario. } \\
\text { Describirán los efectos } \\
\text { adversos derivados } \\
\text { del incumplimiento } \\
\text { farmacológico }\end{array}$ & $\begin{array}{l}\text { Objetivo: } \\
\text { Alcanzado } \\
\text { No alcanzado } \\
\text { Firma: }\end{array}$ \\
\hline
\end{tabular}

